\title{
CHARACTERIZATIONS OF STRONG WELL-POSEDNESS FOR A CLASS OF MULTI-VALUED VARIATIONAL INEQUALITIES
}

\author{
M. OVEISIHA
}

Received 03 June, 2016

\begin{abstract}
In this paper, by using the limiting subdifferential we consider the well-posedness for multi-valued variational inequalities and give some equivalence formulations for them. Moreover, we show that the strong well-posedness for a multi-valued variational inequality is equivalent to the existence and uniqueness of its solution.
\end{abstract}

2010 Mathematics Subject Classification: 49K40; 49J52; 90C31

Keywords: limiting subdifferential, variational inequality, well-posedness, approximating sequence

\section{INTRODUCTION}

The classical concept of well-posedness for a minimization problem, which has been known as the Tykhonov well-posedness, was introduced by Tykhonov [7] in 1966. A minimization problem is Tykhonov well-posed if it has a unique solution and every minimizing sequence of the problem converges to the unique solution. In the last decades, various concepts of well-posedness such as $\alpha$-well-posedness, Hadamard well-posedness, Levitin-Polyak well-posedness and well-posedness by perturbations have been presented and studied for optimization problems, see [3, 4, 10, 12] and references therein. The concept of well-posedness for hemivariational inequality was first introduced by Goeleven and Mentagui [2] to provide some conditions guaranteing the existence and uniqueness of a solution for a hemivariational inequality. Later, Xiao and Huang [10] considered a concept of well-posedness for a variationalhemivariational inequality and obtained the equivalence of well-posedness between the variational-hemivariational inequality and the corresponding inclusion problem. Very recently, Xiao et al. [9] established two kinds of conditions under which the strong and weak well-posedness for the hemivariational inequality are equivalent to the existence and uniqueness of its solutions, respectively.

In this paper, by using the limiting subdifferential we extend the concept of wellposedness to a class of multi-valued variational inequality which include as a special 
case the classical variational and hemivariational inequalities. Moreover, we establish some equivalence results for them. The paper is organized as follows: Section 2 prepares briefly some preliminary notions and results used in sequel. In Section 3, we show that the strong well-posedness for a multi-valued variational inequality is equivalent to the existence and uniqueness of its solution. Also, a metric characterization for the strong well-posedness of multi-valued variational inequality is obtained.

\section{NOTATIONS AND PRELIMINARIES}

Let $X$ be a Banach space and $X^{*}$ its topological dual space. The norm in $X$ and $X^{*}$ will be denoted by $\|$.$\| . We denote \langle.,\rangle,.[x, y]$ and $] x, y[$ the dual pair between $X$ and $X^{*}$, the line segment for $x, y \in X$, and the interior of $[x, y]$, respectively. Now, we recall some concepts of subdifferentials that we need in the next section.

Definition 1 ([6]). Let $X$ be a normed vector space, $\Omega$ be a nonempty subset of $X, x \in \Omega$ and $\varepsilon \geq 0$. The set of $\varepsilon$-normals to $\Omega$ at $x$ is

$$
\widehat{N}_{\varepsilon}(x ; \Omega):=\left\{x^{*} \in X^{*} \mid \limsup _{u \rightarrow x} \frac{\left\langle x^{*}, u-x\right\rangle}{\|u-x\|} \leq \varepsilon\right\} .
$$

Assume that $\bar{x} \in \Omega$, the limiting normal cone to $\Omega$ at $\bar{x}$ is

$$
N(\bar{x} ; \Omega):=\limsup _{x \rightarrow \bar{x}, \varepsilon \downarrow 0} \widehat{N}_{\varepsilon}(x ; \Omega) .
$$

Let $J: X \rightarrow \overline{\mathbb{R}}$ be finite at $\bar{x} \in X$; the limiting subdifferential of $J$ at $\bar{x}$ is defined as follows

$$
\partial_{M} J(\bar{x}):=\left\{x^{*} \in X^{*} \mid\left(x^{*},-1\right) \in N((\bar{x}, J(\bar{x})) ; \text { epi } J)\right\} .
$$

Remark 1 ([6]). The set-valued mapping $x \mapsto \partial_{M} J(x)$ has closed graph for locally Lipschitz functions.

Definition 2. Let $T: X \rightarrow 2^{X^{*}}$ be a set-valued mapping. $T$ is said to be relaxed invariant monotone with respect to $\eta$ if there exists a constant $\alpha$ such that for any $x, y \in X$ and any $u \in T(x), v \in T(y)$, one has

$$
\langle v, \eta(x, y)\rangle+\langle u, \eta(y, x)\rangle \leq-\alpha\left(\|\eta(x, y)\|^{2}+\|\eta(y, x)\|^{2}\right) .
$$

Remark 2. (1) When $T: X \rightarrow X^{*}$ is a single-valued operator, we obtain the definition of a relaxed invariant monotone operator.

(2) If $\alpha=0$, then the Definition 2 reduces to the definition of an invariant monotone map.

Definition 3 ([11]). A mapping $T: X \rightarrow X^{*}$ is said to be hemicontinuous if for any $x_{1}, x_{2} \in X$, the function $t \mapsto\left\langle T\left(x_{1}+t x_{2}\right), x_{2}\right\rangle$ from $[0,1]$ into $]-\infty,+\infty[$ is continuous at $0_{+}$.

Condition $C$ ([5]). Let $\eta: X \times X \rightarrow X$. Then, for any $x, y \in X, \lambda \in[0,1]$

$$
\eta(y, y+\lambda \eta(x, y))=-\lambda \eta(x, y), \quad \eta(x, y+\lambda \eta(x, y))=(1-\lambda) \eta(x, y) .
$$


Remark 3. By some computation, we can see that if Condition $C$ holds, then for any $x_{1}, x_{2} \in X$ and $\lambda \in[0,1]$

$$
\eta\left(x_{1}+\lambda \eta\left(x_{2}, x_{1}\right), x_{1}\right)=\lambda \eta\left(x_{2}, x_{1}\right) .
$$

Now, suppose that $J: X \rightarrow \mathbb{R}, \eta: X \times X \rightarrow X, A: X \rightarrow X^{*}$ is a mapping and $f \in X^{*}$ is some given element. Consider the following multi-valued variational-like inequality associated with $(A, f, J)$ :

$M V L I(A, f, J)$ : Find $\bar{x} \in X$ such that for any $x \in X$, there exists $\xi \in \partial_{M} J(\bar{x})$, that

$$
\langle A \bar{x}-f+\xi, \eta(x, \bar{x})\rangle \geq 0 .
$$

Definition 4. A sequence $\left\{x_{n}\right\} \subset X$ is said to be an approximating sequence for the $\operatorname{MVLI}(A, f, J)$, if there exists $\left\{\epsilon_{n}\right\}$ with $\epsilon_{n} \downarrow 0$ such that for any $x \in X$ there exists $x_{n}^{*} \in \partial_{M} J\left(x_{n}\right)$, that

$$
\left\langle A x_{n}-f+x_{n}^{*}, \eta\left(x, x_{n}\right)\right\rangle \geq-\epsilon_{n}\left\|\eta\left(x, x_{n}\right)\right\| .
$$

Definition 5. The multi-valued variational-like inequality $M V L I(A, f, J)$ is said to be strongly well-posed if it has a unique solution $\bar{x}$ on $X$ and for every approximating sequence $\left\{x_{n}\right\}, \eta\left(\bar{x}, x_{n}\right)$ converges strongly to 0 .

\section{MAIN RESULTS}

In this section, we establish some conditions under which the well-posedness for the multi-valued variational-like inequality is equivalent to the existence and uniqueness of its solution. Theorems in this section extend theorems in $[8,9]$ from hemivariational inequalities with Clarke's subdifferential which is convex to multi-valued variational-like inequalities with limiting subdifferential which is not necessarily convex.

Theorem 1. Assume that operator $A: X \rightarrow X^{*}$ is hemicontinuous and relaxed invariant monotone with constant $c$ and $J$ is locally Lipschitz such that $\partial_{M} J$ satisfies relaxed invariant monotonicity condition with constant $\alpha$. Consider the following assertions:

(i) $\bar{x}$ is a solution of the $\operatorname{MVLI}(A, f, J)$.

(ii) $\bar{x}$ is a solution of the following associated multi-valued variational-like inequality:

$A M \operatorname{VLI}(A, f, J):$ Find $\bar{x} \in X$ such that for any $x \in X$ there exists $x^{*} \in$ $\partial_{M} J(x)$ such that $\left\langle A x-f+x^{*}, \eta(x, \bar{x})\right\rangle \geq 0$.

If $c+\alpha \geq 0$ and $\eta$ is skew, then $(i) \Rightarrow(i i)$. If $\eta$ satisfies Condition $C$, then $(i i) \Rightarrow(i)$.

Proof. $(i) \Rightarrow(i i)$. Let $\bar{x} \in X$ be a solution of $\operatorname{MVLI}(A, f, J)$. Hence, for any $x \in X$ there exists $\xi \in \partial_{M} J(\bar{x})$ such that

$$
\langle A \bar{x}-f+\xi, \eta(x, \bar{x})\rangle \geq 0 .
$$


By the relaxed invariant monotonicity of $\partial_{M} J$, for any $x \in X$ and $x^{*} \in \partial_{M} J(x)$, one has

$$
\left\langle x^{*}, \eta(\bar{x}, x)\right\rangle+\langle\xi, \eta(x, \bar{x})\rangle \leq-\alpha\left(\|\eta(x, \bar{x})\|^{2}+\|\eta(\bar{x}, x)\|^{2}\right) .
$$

It follows from relaxed invariant monotonicity of the operator $A,(3.1)$ and (3.2), that

$$
\begin{aligned}
\left\langle A x-f+x^{*},\right. & \eta(x, \bar{x})\rangle \geq\left\langle A x+x^{*}, \eta(x, \bar{x})\right\rangle-\langle A \bar{x}+\xi, \eta(x, \bar{x})\rangle \\
& =-\left[\langle A x, \eta(\bar{x}, x)\rangle+\langle A \bar{x}, \eta(x, \bar{x})\rangle+\left\langle x^{*}, \eta(\bar{x}, x)\right\rangle+\langle\xi, \eta(x, \bar{x})\rangle\right] \\
& \geq(c+\alpha)\left(\|\eta(x, \bar{x})\|^{2}+\|\eta(\bar{x}, x)\|^{2}\right),
\end{aligned}
$$

which shows that $\bar{x}$ is a solution of $A M V L I(A, f, J)$.

$(i i) \Rightarrow(i)$. Conversely, let $\bar{x}$ be a solution to the $A M V L I(A, f, J)$. Hence, for any $x \in X$ there exists $x^{*} \in \partial_{M} J(x)$ such that

$$
\left\langle A x-f+x^{*}, \eta(x, \bar{x})\right\rangle \geq 0 .
$$

For any $z \in X$ and $t \in[0,1]$, set $x(t)=\bar{x}+t \eta(z, \bar{x})$ in inequality (3.3), we have

$$
\left\langle A(\bar{x}+t \eta(z, \bar{x}))-f+x_{t}^{*}, \eta(\bar{x}+t \eta(z, \bar{x}), \bar{x})\right\rangle \geq 0,
$$

that $x_{t}^{*} \in \partial_{M} J(x(t))$. It follows Condition $\mathrm{C}$, that

$$
\left\langle A(\bar{x}+t \eta(z, \bar{x}))-f+x_{t}^{*}, \eta(z, \bar{x})\right\rangle \geq 0 .
$$

Since $J$ is locally Lipschitz, we deduce that $\partial_{M} J$ is locally bounded (Corollary 1.81 in [6]). Hence, there exists a neighborhood of $\bar{x}$ and a constant $\ell>0$ such that for each $z$ in this neighborhood and $x^{*} \in \partial_{M} J(z)$, we have $\left\|x^{*}\right\| \leq \ell$. Since $x(t) \rightarrow \bar{x}$ when $t \rightarrow 0$ for $t$ to be sufficiently small $\left\|x_{t}^{*}\right\| \leq \ell$, without loss of generality we may assume that $x_{t}^{*} \rightarrow x^{*}$ in weak ${ }^{*}$-topology. Now, hemicontinuity of the operator $A$ on $X$ implies that

$$
\left\langle A \bar{x}-f+x^{*}, \eta(z, \bar{x})\right\rangle \geq 0 .
$$

By the arbitrariness of $z \in X$, we conclude that $\bar{x}$ is a solution of $\operatorname{MVLI}(A, f, J)$.

Proposition 1. Let $C^{*} \subset X^{*}$ be nonempty, closed, convex and bounded, $\varphi: X \rightarrow$ $\mathbb{R}$ be proper, convex and lower semi-continuous and $y \in X$ be arbitrary. Assume that $\eta$ is continuous and affine with respect to the first argument and for each $x \in X$, there exists $x^{*}(x) \in C^{*}$ such that

$$
\left\langle x^{*}(x), \eta(x, y)\right\rangle \geq \varphi(y)-\varphi(y+\eta(x, y)) .
$$

Then, there exists $y^{*} \in C^{*}$ such that

$$
\left\langle y^{*}, \eta(x, y)\right\rangle \geq \varphi(y)-\varphi(y+\eta(x, y)), \quad \forall x \in X .
$$

Proof. With some minor modification in the proof of Proposition 3.3 in [1], we can deduce the proof. 
Theorem 2. Let $A: X \rightarrow X^{*}$ be relaxed invariant monotone with constant $c$ and $J$ a l.s.c. function that its limiting subdifferential satisfies relaxed invariant monotonicity condition with constant $\alpha$. If $\alpha+c>0$ and $\eta$ is continuous and affine with respect to the second argument and skew, then $M V L I(A, f, J)$ is strongly well-posed if and only if it has a unique solution on $X$.

Proof. The necessity is obvious. For the sufficiency, suppose that $\operatorname{MVLI}(A, f, J)$ has a unique solution $\bar{x}$. Since $\bar{x}$ is the unique solution of $\operatorname{MVLI}(A, f, J)$, for any $x \in X$, there exists $x^{*} \in \partial_{M} J(\bar{x})$ such that

$$
\left\langle A \bar{x}-f+x^{*}, \eta(x, \bar{x})\right\rangle \geq 0 .
$$

Suppose that $\left\{x_{n}\right\}$ is an approximating sequence for $\operatorname{MVLI}(A, f, J)$. It follows that there exists $\epsilon_{n} \downarrow 0$ such that for any $x \in X$, there exists $\xi_{n}(x) \in \partial_{M} J\left(x_{n}\right)$ that

$$
\left\langle A x_{n}-f+\xi_{n}(x), \eta\left(x, x_{n}\right)\right\rangle \geq-\epsilon_{n}\left\|\eta\left(x, x_{n}\right)\right\| .
$$

Now, consider the nonempty, convex and bounded set $\operatorname{co}\left\{A x_{n}-f+\xi_{n} \mid \xi_{n} \in \partial_{M} J\left(x_{n}\right)\right\}$. Hence, it follows from Proposition 1 with $\varphi(x)=\epsilon_{n}\left\|x-x_{n}\right\|$ that there exists $\xi_{n}$ which is independent on $x$, such that

$$
\left\langle A x_{n}-f+\xi_{n}, \eta\left(x, x_{n}\right)\right\rangle \geq-\epsilon_{n}\left\|\eta\left(x, x_{n}\right)\right\|, \quad \forall x \in X .
$$

By choosing $\xi_{n}$, we can set $\xi_{n}=\sum_{i=1}^{m} \lambda_{i} \xi_{n}^{i}$ that $m \in \mathbb{N}, \sum_{i=1}^{m} \lambda_{i}=1$ and $\xi_{n}^{i} \in$ $\partial_{M} J\left(x_{n}\right)$. Hence,

$$
\sum_{i=1}^{m} \lambda_{i}\left\langle A x_{n}-f+\xi_{n}^{i}, \eta\left(x, x_{n}\right)\right\rangle \geq-\epsilon_{n}\left\|\eta\left(x, x_{n}\right)\right\|, \quad \forall x \in X .
$$

Now, set $x=\bar{x}$ in above inequality, yields

$$
\sum_{i=1}^{m} \lambda_{i}\left\langle A x_{n}-f+\xi_{n}^{i}, \eta\left(\bar{x}, x_{n}\right)\right\rangle \geq-\epsilon_{n}\left\|\eta\left(\bar{x}, x_{n}\right)\right\| .
$$

Hence, it follows from relaxed invariant monotonicity of the operator $A$, relaxed invariant monotonicity of the $\partial_{M} J$, the skewness of $\eta$ and above inequality that

$$
\begin{aligned}
-\epsilon_{n}\left\|\eta\left(\bar{x}, x_{n}\right)\right\| & \leq \sum_{i=1}^{m} \lambda_{i}\left\langle A x_{n}-f+\xi_{n}^{i}, \eta\left(\bar{x}, x_{n}\right)\right\rangle \\
& \leq \sum_{i=1}^{m} \lambda_{i}\left[\left\langle A x_{n}+\xi_{n}^{i}, \eta\left(\bar{x}, x_{n}\right)\right\rangle-\left\langle A \bar{x}+\zeta_{n}^{i}, \eta\left(\bar{x}, x_{n}\right)\right\rangle\right] \\
& =\sum_{i=1}^{m} \lambda_{i}\left[-\left\langle A \bar{x}-A x_{n}+\zeta_{n}^{i}-\xi_{n}^{i}, \eta\left(\bar{x}, x_{n}\right)\right\rangle\right. \\
& \leq-\sum_{i=1}^{m} 2 \lambda_{i}(c+\alpha)\left\|\eta\left(\bar{x}, x_{n}\right)\right\|^{2}=-2(c+\alpha)\left\|\eta\left(\bar{x}, x_{n}\right)\right\|^{2},
\end{aligned}
$$


where $\zeta_{n}^{i} \in \partial_{M} J(\bar{x})$ is obtained from (3.4) by setting $x=x_{n}$. Since $c+\alpha>0$, it follows that

$$
\left\|\eta\left(\bar{x}, x_{n}\right)\right\| \leq \frac{\epsilon_{n}}{2(c+\alpha)} .
$$

Taking limit at both sides of the above inequality, implies that $\eta\left(\bar{x}, x_{n}\right)$ converges strongly to 0 .

Example 1. Let $X=\mathbb{R}, f=0, A$ be the identity map and $J$ be defined as

$$
J(x)=\left\{\begin{array}{cl}
x^{2}+2 x & \text { if } x>0 \\
x^{2}-x & \text { if } x \leq 0
\end{array}\right.
$$

The limiting subdifferential of $J$ is

$$
\partial_{M} J(x)=\left\{\begin{array}{cc}
2 x+2 & \text { if } x>0, \\
{[-1,2]} & \text { if } x=0, \\
2 x-1 & \text { if } x<0 .
\end{array}\right.
$$

Let $\eta$ be defined as $\eta(x, y):=k(x-y)$, such that $0<k \leq 1$. Then by some computation we can see that $\partial_{M} J, A$ are relaxed invariant monotone with constants $\alpha=1$, $c=\frac{1}{2}$, respectively. Hence, all assumptions of Theorem 2 are fulfilled and $\bar{x}=0$ is a unique solution of (MVLI) and therefore it is strongly well-posed.

For any $\epsilon>0$, consider the following two sets:

$$
\begin{array}{r}
\Omega(\epsilon)=\left\{\bar{x}: \forall x \in X, \exists x^{*} \in \partial_{M} J(\bar{x}) \text { s.t. }\left\langle A \bar{x}-f+x^{*}, \eta(x, \bar{x})\right\rangle \geq-\epsilon\|\eta(x, \bar{x})\|\right\}, \\
\Psi(\epsilon)=\left\{\bar{x}: \forall x \in X, \quad \exists x^{*} \in \partial_{M} J(\bar{x}) \text { s.t. }\langle-A x, \eta(\bar{x}, x)\rangle+\left\langle-f+x^{*}, \eta(x, \bar{x})\right\rangle \geq\right. \\
-\epsilon\|\eta(x, \bar{x})\|\} .
\end{array}
$$

Lemma 1. Suppose that $A: X \rightarrow X^{*}$ is invariant monotone and hemicontinuous. Then $\Omega(\epsilon)=\Psi(\epsilon)$ for all $\epsilon>0$.

Proof. Taking into account the invariant monotonicity of mapping $A$, it is easy to obtain that $\Omega(\epsilon) \subset \Psi(\epsilon)$. For the other side suppose that $\bar{x} \in \Psi(\epsilon)$. Then, for any $x \in X$, there exists $x^{*} \in \partial_{M} J(\bar{x})$ such that

$$
\langle-A x, \eta(\bar{x}, x)\rangle+\left\langle-f+x^{*}, \eta(x, \bar{x})\right\rangle \geq-\epsilon\|\eta(x, \bar{x})\| .
$$

Set $x=\bar{x}+t \eta(z, \bar{x})$ in (3.5), that $z \in X$ and $t \in[0,1]$, yields

$$
\begin{gathered}
\langle-A(\bar{x}+t \eta(z, \bar{x})), \eta(\bar{x}, \bar{x}+t \eta(z, \bar{x}))\rangle+\left\langle-f+x^{*}, \eta(\bar{x}+t \eta(z, \bar{x}), \bar{x})\right\rangle \geq \\
-\epsilon\|\eta(\bar{x}+t \eta(z, \bar{x}), \bar{x})\| .
\end{gathered}
$$

By using Condition $\mathrm{C}$, we obtain

$$
\langle-A(\bar{x}+t \eta(z, \bar{x})),-\eta(z, \bar{x})\rangle+\left\langle-f+x^{*}, \eta(z, \bar{x})\right\rangle \geq-\epsilon\|\eta(z, \bar{x})\| .
$$

Now, it follows from the hemicontinuity of mapping $A$ that

$$
\left\langle A \bar{x}-f+x^{*}, \eta(z, \bar{x})\right\rangle \geq-\epsilon\|\eta(z, \bar{x})\|,
$$

which shows that $\bar{x} \in \Omega(\epsilon)$. This completes the proof. 
Lemma 2. Suppose that $A: X \rightarrow X^{*}$ is a hemicontinuous mapping. If $J$ is locally Lipschitz and $\eta$ is continuous with respect to the second argument, then $\Omega(\epsilon)$ is closed in $X$ for all $\epsilon>0$.

Proof. Let $\left\{x_{n}\right\} \subset \Omega(\epsilon)$ be a sequence such that $x_{n} \rightarrow \bar{x}$ in $X$. Then for any $x \in X$, there exists $x_{n}^{*} \in \partial_{M} J\left(x_{n}\right)$ such that

$$
\left\langle A x_{n}-f+x_{n}^{*}, \eta\left(x, x_{n}\right)\right\rangle \geq-\epsilon\left\|\eta\left(x, x_{n}\right)\right\| .
$$

Since $J$ is locally Lipschitz, there exists a subsequence of $x_{n}^{*}$ that convergent to a $x^{*} \in \partial_{M} J(\bar{x})$ in weak*-topology. Consider (3.6) with this subsequence, taking limit at both sides of it and using this fact that $A$ is hemicontinuous and $\eta$ is continuous with respect to the second argument, we obtain

$$
\left\langle A \bar{x}-f+x^{*}, \eta(x, \bar{x})\right\rangle \geq-\epsilon\|\eta(x, \bar{x})\|,
$$

which implies that $\bar{x} \in \Omega(\epsilon)$. This completes the proof.

Theorem 3. Suppose that $A: X \rightarrow X^{*}$ is hemicontinuous and invariant monotone with respect to $\eta$ that $\eta$ is continuous with respect to the second argument, satisfies Condition $C$ and skew. Then $M V L I(A, f, J)$ is strongly well-posed if and only if

$$
\Omega(\epsilon) \neq \varnothing, \forall \epsilon>0 \quad \text { and } \quad \operatorname{diam}(\Omega(\epsilon)) \rightarrow 0 \quad \text { as } \quad \epsilon \rightarrow 0 .
$$

Proof. "Necessity" follows similarly from the first part of Theorem 3.1 in [8]. Hence, we prove the "Sufficiency". Suppose that $\left\{x_{n}\right\} \subset X$ is an approximating sequence for $\operatorname{MVLI}(A, f, J)$. Then there exist a nonnegative sequence $\epsilon_{n} \rightarrow 0$ such that for any $x \in X$, there exists $\xi_{n} \in \partial_{M} J\left(x_{n}\right)$ such that

$$
\left\langle A x_{n}-f+\xi_{n}, \eta\left(x, x_{n}\right)\right\rangle \geq-\epsilon_{n}\left\|\eta\left(x, x_{n}\right)\right\|,
$$

it means that $x_{n} \in \Omega\left(\epsilon_{n}\right)$. It follows from $\operatorname{diam}(\Omega(\epsilon)) \rightarrow 0$, that $\left\{x_{n}\right\}$ is a cauchy sequence and so converges strongly to some point $\bar{x} \in X$. Since $J$ is locally lipschitz, there exists a subsequence of $\xi_{n}$ (e.g. $\left.\left\{\xi_{n_{i}}\right\}\right)$ that is convergent to a $\xi \in \partial_{M} J(\bar{x})$ in weak*-topology. Taking limit at the both side of (3.7) and using this fact that $A$ is monotone and $\eta$ is skew and continuous with respect to the second argument, we obtain

$$
\begin{aligned}
\langle A x-f+\xi, \eta(x, \bar{x})\rangle & =\lim _{i \rightarrow \infty}\left\langle A x-f+\xi_{n_{i}}, \eta\left(x, x_{n_{i}}\right)\right\rangle \\
& \geq \lim _{i \rightarrow \infty}\left\langle A x_{n_{i}}-f+\xi_{n_{i}}, \eta\left(x, x_{n_{i}}\right)\right\rangle \\
& \geq \lim _{i \rightarrow \infty}-\epsilon_{n_{i}}\left\|\eta\left(x, x_{n_{i}}\right)\right\|=0 .
\end{aligned}
$$

Now, by using Theorem $1, \bar{x}$ is a solution of $\operatorname{MVLI}(A, f, J)$. Since, $\operatorname{diam}(\Omega(\epsilon)) \rightarrow$ 0 when $\epsilon \rightarrow 0, \operatorname{MVLI}(A, f, J)$ has a unique solution. This completes the proof. 


\section{REFERENCES}

[1] F. Giannessi and A. Khan, "Regularization of non-coercive quasi variational inequalities," Control Cyber., vol. 29, pp. $91-110,2000$.

[2] D. Goeleven and D. Mentagui, "Well-posed hemivariational inequalities," Numer. Funct. Anal. Optim., vol. 16, pp. $909-921,1995$.

[3] X. X. Huang, X. Q. Yang, and D. L. Zhu, "Levitin-polyak well-posedness of variational inequality problems with functional constraints," J. Global Optim., vol. 44, pp. 159 - 174, 2009, doi: 10.1007/s10898-008-9310-1.

[4] X. J. Long and N. J. Huang, "Metric characterizations of $\alpha$-well-posedness for symmetric quasiequilibrium problems," J. Global Optim., vol. 45, pp. 459 - 471, 2009, doi: 10.1007/s10898-0089385-8.

[5] S. R. Mohan and S. K. Neogy, "On invex sets and preinvex functions," J. Math. Anal. Appl., vol. 189, pp. 901 - 908, 1995, doi: 10.1006/jmaa.1995.1057.

[6] B. S. Mordukhovich, Variational Analysis and Generalized Differential I, Basic theory, 1st ed., ser. Grundlehren. Berlin: Springer Berlin Heidelberg, 2006, vol. 330.

[7] A. N. Tikhonov, "On the stability of the functional optimization problem," Comput. Math. Math. Phys., vol. 6, pp. $28-33,1966$.

[8] Y. B. Xiao, N. J. Huang, and M. M. Wong, "Well-posedness of hemivariational inequalities and inclusion problems," Taiwanese J. Math., vol. 15, pp. 1261 - 1276, 2011.

[9] Y. B. Xiao, X. Yang, and N. J. Huang, "Some equivalence results for well-posedness of hemivariational inequalities," J. Global Optim., vol. 61, pp. 789 - 802, 2015, doi: 10.1007/s10898-0140198-7.

[10] Y. Xiao and N. Huang, "Well-posedness for a class of variational-hemivariational inequalities with perturbations," J. Optim. Theory Appl., vol. 151, pp. 33 - 51, 2011, doi: 10.1007/s10957011-9872-9.

[11] E. Zeidler, Nonlinear Functional Analysis and its Applications. Berlin: Springer Berlin Heidelberg, 1990, vol. II.

[12] J. Zeng, S. J. Li, W. Y. Zhang, and X. W. Xue, "Hadamard well-posedness for a set-valued optimization problem," Optim. Lett., vol. 7, pp. 559 - 573, 2013, doi: 10.1007/s11590-011-0439-3.

Author's address

M. Oveisiha

Department of Mathematics, Faculty of Science, Imam Khomeini International University, P.O. Box 34149-16818, Qazvin, Iran

E-mail address: oveisiha@sci.ikiu.ac.ir 